TRANSACTIONS OF THE

AMERICAN MATHEMATICAL SOCIETY

Volume 356, Number 10, Pages 4143-4159

S 0002-9947(04)03457-9

Article electronically published on April 16, 2004

\title{
ON THE CLASSIFICATION OF FULL FACTORS OF TYPE III
}

\author{
DIMITRI SHLYAKHTENKO
}

\begin{abstract}
We introduce a new invariant $\mathscr{S}(M)$ for type III factors $M$ with no almost-periodic weights. We compute this invariant for certain free ArakiWoods factors. We show that Connes' invariant $\tau$ cannot distinguish all isomorphism classes of free Araki-Woods factors. We show that there exists a continuum of mutually non-isomorphic free Araki-Woods factors, each without almost-periodic weights.
\end{abstract}

\section{INTRODUCTION}

The necessity to find effective invariants to distinguish full type III factors comes from the problem of classifying type III (typically, type $\mathrm{III}_{1}$ ) factors naturally occurring in free probability theory of Voiculescu [22. These factors arise as free products of finite-dimensional or hyperfinite von Neumann algebras 1, 14, 13, 6 and more generally from a second-quantization procedure involving the free Gaussian functor (the so-called free Araki-Woods factors, 15]). The classification results so far have all relied on Connes' $\mathrm{S}$ and Sd invariants [3] [4, and worked well for factors having almost periodic weights (for example, in [15] a complete classification of free Araki-Woods factors for which the free quasi-free state is almost-periodic was given). However, not all free Araki-Woods factors have almost periodic weights [17, and the question of their complete classification remains open.

Recall that given a factor $M$ and a state $\phi$ on $M$, Tomita-Takesaki theory associates to it a one-parameter group of automorphisms $\sigma_{t}^{\phi}, t \in \mathbb{R}$, known as the modular group. If $\sigma_{t}^{\phi}$ is not inner for all $t$, then $M$ is a type III factor. Our case of interest is the situation that $\sigma_{t}^{\phi}$ is never inner (for $t \neq 0$ ), as is the case for factors of type $\mathrm{III}_{1}$. The modular group $\sigma_{t}^{\phi}$ depends on $\phi$ only up to inner automorphisms, thanks to the Connes Radon-Nikodym type theorem. The importance of the modular group $\sigma_{t}^{\phi}$ is even more apparent from the fact that the crossed product $M \rtimes_{\sigma^{\phi}} \mathbb{R}$ is semi-finite (and is a factor of type $\mathrm{II}_{\infty}$ if $M$ is type $\mathrm{III}_{1}$ ). This crossed product is independent of the choice of $\phi$, and is called the core of $M$.

It is fair to say that most invariants of type III factors arise in one of two ways: either through the analysis of the modular group (such as its spectral properties or periodicity, as in Connes' $S$ and $T$ invariants introduced in [3]), or the analysis of the core, to which some of the properties of the factor pass (e.g., injectivity).

1.1. Modular invariants. We will briefly review the invariants of a type $\mathrm{III}_{1}$ factor that can be obtained from the modular group; most of these constructions are from Connes' paper [4].

Received by the editors July 21, 2002 and, in revised form, July 17, 2003.

2000 Mathematics Subject Classification. Primary 46L10; Secondary 46L54. 
Although $\sigma_{t}^{\phi}$ depends on $\phi$ only up to inner automorphisms, the degree of continuity of $t \mapsto \sigma_{t}^{\phi}$ may vary from one state to another. To give a trivial example, let $N$ be a type $\mathrm{II}_{1}$ factor with a trace $\tau$, and consider the state $\phi: N \rightarrow \mathbb{R}$ given by $\phi(x)=\tau\left(d^{1 / 2} x d^{1 / 2}\right)$. The modular group associated to $\phi$ is given by $x \mapsto d^{i t} x d^{-i t}$. If $d=1$, so that its spectrum is the set $\{1\}$, this map is continuous with respect to any topology on $\mathbb{R}$. On the other hand, if the spectral measure of $d$ is absolutely continuous with respect to Lebesgue measure, then this map is (say, *-strongly) continuous if and only if $\mathbb{R}$ is endowed with the usual topology. An intermediate situation occurs when the spectral measure of $d$ is atomic. In this case, the action is continuous with respect to a certain topology $\tau$, for which the completion of $\mathbb{R}$ is a compact topological group $T$ (the inclusion $\mathbb{R} \subset T$ is dual to the inclusion of the group generated by the atoms of the spectral measure of $d$ into $\mathbb{R}_{+}$, the multiplicative group of positive reals).

Utilizing this idea, Connes has introduced the invariant $\tau(M)$, which is, roughly, the weakest topology on $\mathbb{R}$ making the modular group continuous (modulo inner automorphisms); see below for a precise definition.

In the case that for some state $\phi$ the modular group $\sigma_{t}^{\phi}$ is continuous with respect to to some topology $\tau$, for which the completion of $\mathbb{R}$ is a locally compact group $G$, there arises a possibility to consider the $G$-core of $M$, using the extension of the action of the modular group from $\mathbb{R}$ to $G$. It is interesting to note that this core now depends on the choice of $\phi$ (since not every choice of $\phi$ will lead to the same degree of continuity). This in turn can be used to select "special" states on $M$. It turns out that there are only two situations, insisting that $G$ is locally compact: either $G$ is all of $\mathbb{R}$ (so that $\tau$ is the usual topology), or $G$ is a compact group (necessarily a torus, i.e. a finite or infinite power of the circle group), and $R \subset G$ is an "irrational line" embedding of $\mathbb{R}$ into the appropriate torus. If the latter is the case, the state $\phi$ must be "almost-periodic" (in that the modular operator associated to $\phi$ has atomic spectral measure). It turns out that in certain cases, the "smallest" $G$-core (corresponding to the "most continuous" $\phi$ and the largest $G$ ) can be characterized. More precisely, it turns out that if the $G$-core of $M$ is non- $\Gamma$, then $G$ must be maximal (for no state $\phi$ on $M$ can the modular group extend by continuity to a group larger than $G$ ).

It would be very interesting to understand if some analog of the $G$-core construction can be carried out for non-locally compact groups.

1.2. Absence of almost-periodic states. Almost-periodic states (or even weights) do not always exist. Much of this paper is devoted to the exploration of free Araki-Woods factors, having no almost-periodic states or weights. We give an example of a one-parameter family of such factors, each having no almost-periodic states (or even weights), but with different $\tau$-invariants (and hence pairwise nonisomorphic).

It turns out, however, that the $\tau$ invariant is insufficient to classify free ArakiWoods factors.

We introduce a new invariant for a factor $M$, given by the intersection over the set of all normal faithful states $\phi$ on $M$ of the collections of measures, absolutely continuous with respect to the spectral measure of the modular operator of $\phi$. This invariant is in spirit related to the Connes' Sd invariant, where the intersection is taken over all almost-periodic weights on $M$. Amazingly, it turns out that our invariant can be computed for certain free Araki-Woods factors. Using our new 
invariant we are able to produce a pair of non-isomorphic free Araki-Woods factors, which cannot be distinguished by their $\tau$ invariant, i.e., cannot be distinguished by any previously known invariant for type III factors.

The idea of the computation lies in the remark that the $\mathbb{R}$-core of $M$ must have a special abelian subalgebra, namely $L(\mathbb{R}) \subset M \rtimes G$. However, Voiculescu showed that there are sometimes restrictions on the kinds of abelian subalgebras that can exist inside semifinite von Neumann algebras. For example, he showed that free group factors $N=L\left(\mathbb{F}_{n}\right)$ cannot have diffuse abelian subalgebras $A$ for which $N$, when regarded as an $A, A$-bimodule, is "disjoint" from the coarse $A, A$-bimodule $A \bar{\otimes} A$. More generally, the same statement holds for von Neumann algebras having a set of generators with large free entropy dimension. Translating the restriction on the possible subalgebras $A$ back to the subalgebra $L(\mathbb{R}) \subset M \rtimes G$ produces a restriction on the spectral properties of the action. It turns out that, under suitable assumptions, the spectral measure associated to the action of $\mathbb{R}$ cannot be disjoint from the Haar measure on the group. This in turn implies a restriction on the possible spectral measures of states on $M$.

\section{Some EXAmples of topologies ASSOCiated TO UNITARY REPRESENTATIONS OF $\mathbb{R}$}

The purpose of this section is to set notation and to prove certain results about unitary representation of $\mathbb{R}$, which are needed in the rest of the paper.

2.1. Spectral measures of group representations. Let $\mu$ be a measure on a measure space $X$. Denote by $\mathscr{C}_{\mu}$ the collection of measures on $X$

$$
\mathscr{C}_{\mu}=\{\nu: \mu(Y)=0 \Rightarrow \nu(Y)=0 \text { for all measurable } Y \subset X\} .
$$

In other words, $\mathscr{C}_{\mu}$ is the collection of all measures, which are absolutely continuous with respect to $\mu$. We shall abuse notation and write $\mathscr{C}_{T}$ if $T$ is a self-adjoint operator. By this we mean the collection of all measures, absolutely continuous with respect to the spectral measure of $T$ on $\mathbb{R}$.

It should be mentioned that, following an idea of Mackey, $\mathscr{C}_{\mu}$ can be viewed as a kind of replacement for the notion of the support of $\mu$. Indeed, any $\nu \in \mathscr{C}_{\mu}$ can be written as $f \cdot \mu$ for some function $f$, which is completely determined except on a set of $\mu$-measure zero. One can form intersections of two families $\mathscr{C}_{\mu}$ and $\mathscr{C}_{\nu}$; this operation is to remind us of the notion of intersection of sets. Similarly, inclusion of $\mathscr{C}_{\mu}$ and $\mathscr{C}_{\nu}$ can be thought of as the inclusion of the support of $\mu$ into that of $\nu$. We shall say that a collection of measures $\mathscr{C}$ is supported on a set $Y$ if for all $\nu \in \mathscr{C}$, the complement of $Y$ has measure zero. Note also that if $\mu$ is atomic, then the knowledge of $\mathscr{C}_{\mu}$ is exactly equivalent to the knowledge of the set of atoms of $\mu$.

We now recall some basic facts about representations and duality of locally compact abelian groups (see e.g. [10]). Let $\sigma: G \rightarrow U(H)$ be a $*$-strongly continuous representation of $G$ on a Hilbert space $H$. Associated to $\sigma$ it is spectral measure class $\mathscr{C}_{\sigma}$ on the dual group $\hat{G}$. The class $C_{\sigma}$ can be defined as the smallest collection of measures, so that (1) if $\mu \in \mathscr{C}_{\sigma}$ and $\mu^{\prime}$ is a.c. with respect to $\mu$, then $\mu^{\prime} \in \mathscr{C}_{\sigma}$ and (2) for each $\xi \in H$, the measure obtained as the Fourier transform of the positivedefinite function $g \mapsto\langle\xi, \sigma(g) \xi\rangle$ belongs to $\mathscr{C}_{\sigma}$. If $H$ is separable, there is a measure $\mu$ in $\mathscr{C}_{\sigma}$, with the property that it generates $\mathscr{C}_{\sigma}$ (i.e., $\mathscr{C}_{\sigma}$ is the smallest collection of measures satisfying (1) and containing $\mu$ ). We sometimes refer to this $\mu$ as "the" 
spectral measure of $\sigma$. In particular, $H$ can be decomposed as $H=\int_{\chi \in \hat{G}} H_{\chi} d \mu(\chi)$, so that $\sigma=\int_{\chi \in \hat{G}} \sigma_{\chi}$, where $\sigma_{\chi}(g) \cdot \xi=\chi(g) \cdot \xi, \xi \in H_{\mu}$.

If the group $G$ contains $\mathbb{R}$ as a dense subgroup, the representation $\sigma$ can be restricted to $\mathbb{R} \subset G$. In fact, all representations $\sigma$ of $G$ arise as extensions of representations of $\mathbb{R}$, which are continuous not just in the topology on $\mathbb{R}$, but also in the restriction of the topology $\tau_{G}$ on $G$ to $\mathbb{R} \subset G$. The measure class $\mathscr{C}_{\sigma}$ and the spectral measure $\mu$ of $\sigma$, when interpreted as objects on $\widehat{\mathbb{R}} \supset \hat{G}$, become exactly the measure class and the spectral measure of the restriction of $\sigma$ to $\mathbb{R}$, viewed as a representation of $\mathbb{R}$ (this is evident from the direct integral decomposition formula stated above). In particular, a representation $\pi$ of $\mathbb{R}$ extends to a representation of $G$ iff its spectral measure $\mathscr{C}_{\pi}$ is supported on $\hat{G} \subset \widehat{\mathbb{R}}$.

It is customary to choose a particular spectral measure of a representation of $\mathbb{R}$ on $H$, by finding on $H$ a non-negative operator $A$, for which $\pi(t)=A^{i t}$, and letting $\mu$ be the spectral measure of $A$ (composed with some faithful state on $B(H)$ ). In particular, denoting by $\sigma$ the extension of $\pi$ to $G$, we have $\mathscr{C}_{\sigma}=\mathscr{C}_{\pi}=\mathscr{C}_{\Delta}$.

2.2. Topologies induced by unitary or orthogonal representations of $\mathbb{R}$. Let $\mu$ be a measure on the real line, so that $\mu(-X)=\mu(X)$. Denote by $\pi$ the associated (real or complex) representation of $\mathbb{R}$ on $L^{2}(\mathbb{R}, \mu)$ given by the map

$$
\pi(t)=M_{\exp (2 \pi i t x)},
$$

where $M_{g}$ denotes the operator of multiplication by $g$. Write $\tau(\mu)$ for the weakest topology making the map $t \mapsto \pi(t) \in U\left(L^{2}(\mathbb{R}, \mu)\right)$ continuous with respect to the strong operator topology on the unitary group of $L^{2}(\mathbb{R}, \mu)$. If $\mu$ is not supported on a cyclic subgroup of $\mathbb{R}, \pi$ is injective and $\tau(\mu)$ is a Hausdorff topology.

Proposition 2.1. The completion of $\mathbb{R}$ with respect to the topology $\tau(\mu)$ is a locally compact group iff either $\tau(\mu)$ is the usual topology on $\mathbb{R}$ or $\mu$ is atomic (in which case the completion is compact).

Proof. Denote by $(G, \tau)$ the completion of $(\mathbb{R}, \tau(\mu))$. Then $\mathbb{R} \subset G$ is an inclusion of locally compact abelian groups; by Pontrjagin duality, this inclusion is dual to the injective dense inclusion $\hat{G} \subset \widehat{\mathbb{R}}$.

By the structure theory for locally compact abelian groups [10], the connected component of identity of $\hat{G}$ must have the form $R \times H$, with $R \cong \mathbb{R}^{n}$ and $H$ compact and connected.

First note that $H=\{e\}$. Indeed, the image of $H$ in $\widehat{\mathbb{R}}$ must be a connected compact subgroup of $\widehat{\mathbb{R}}$, hence must be the trivial group.

Since there are no continuous injective maps from $\mathbb{R}^{n}$ into $\mathbb{R}$ for $n>1$, either $n=1$ or $n=0$. If $n=1$, all continuous injective group homomorphisms from $\mathbb{R}$ to itself are surjective (their image must be a path-connected subgroup of $\mathbb{R}$ ). Hence injectivity of $\hat{G} \subset \widehat{\mathbb{R}}$ requires that $\hat{G}=R$ in this case, the inclusion being a homeomorphism onto $\widehat{\mathbb{R}}$ and hence $\tau(\mu)$ being the usual topology on $\mathbb{R}$.

If $n=0, \hat{G}$ must be discrete. This corresponds to the completion $G$ being compact. Moreover, it is not hard to see that $\mu$ must be supported on $\hat{G} \subset \widehat{\mathbb{R}}$, since the representation $\pi$ with spectral measure $\mu$ must extend (by the definition of $\tau(\mu))$ to the completion $G$. Hence $\mu$ is atomic.

Lemma 2.2. A sequence $\left\{t_{n}\right\}_{n=1}^{\infty}$ converges to zero in $\tau(\mu)$ iff $\hat{\mu}\left(t_{n}\right) \rightarrow 1$, where $\hat{\mu}$ is the Fourier transform of $\mu$. 
Proof. Let $\pi$ be a representation of $\mathbb{R}$ associated to $\mu$ as above, and let $\xi \in L^{2}(\mathbb{R}, \mu)$ be the constant function 1 . Then

$$
\hat{\mu}(t)=\langle\pi(t) \xi, \xi\rangle .
$$

By definition, $t_{n} \rightarrow 0$ in $\tau(\mu)$ iff $\pi\left(t_{n}\right) \rightarrow 1$ strongly. The vector state $\phi(T)=$ $\langle T \xi, \xi\rangle$ defines a faithful normal state on the commutative von Neumann algebra $\pi(\mathbb{R})^{\prime \prime} \subset B\left(L^{2}(\mathbb{R}, \mu)\right)$. Hence strong convergence of $\pi\left(t_{n}\right)$ to 1 is equivalent to

$$
\left\|\pi\left(t_{n}\right)-1\right\|_{2}=\phi\left(\left(\pi\left(t_{n}\right)-1\right)\left(\pi\left(t_{n}\right)-1\right)^{*}\right)^{1 / 2} \rightarrow 0 .
$$

In other words, $\pi\left(t_{n}\right) \rightarrow 1$ strongly iff

$$
\frac{1}{2} \phi\left(\pi\left(t_{n}\right)+\pi\left(t_{n}\right)^{*}\right) \rightarrow 1
$$

i.e., $\Re \hat{\mu}\left(t_{n}\right) \rightarrow 1$. Since $\left|\hat{\mu}\left(t_{n}\right)\right| \leq 1$, this happens iff $\hat{\mu}\left(t_{n}\right) \rightarrow 1$.

Theorem 2.3. There exists a continuum of non-atomic measures $\mu_{\lambda}, \lambda \in I$, so that the topologies $\tau\left(\mu_{\lambda}\right)$ are mutually non-equivalent.

Proof. Let $\left\{c_{n}: n=1,2, \ldots\right\}$ be a sequence of real numbers, so that $c_{n} \geq 0$ and $\sum c_{k}^{2}<+\infty$. Denote by $\mu_{n}$ the $n$-fold convolution of delta-measures

$$
\mu_{n}=\left(\frac{1}{2} \delta_{c_{1}}+\frac{1}{2} \delta_{-c_{1}}\right) * \cdots *\left(\frac{1}{2} \delta_{c_{n}}+\frac{1}{2} \delta_{-c_{n}}\right) .
$$

Each $\mu_{n}$ is a symmetric probability measure, and the Fourier transform of $\mu_{n}$ is given by

$$
\hat{\mu}_{n}(t)=\prod_{k=1}^{n} \cos \left(2 \pi c_{k} t\right) .
$$

The Fourier transform of the weak limit $\mu$ of $\mu_{n}$ is given by the pointwise limit of this expression; the measure $\mu$ is non-atomic (see e.g. 8]).

Let $c_{k}=3^{-k !}$. Let $0<\lambda<\frac{1}{2}$ be fixed. Let $t_{n}=\lambda 3^{n !}=\lambda c_{n}^{-1}$. We claim that $t_{n} \rightarrow 0$ in $\tau(\mu)$ iff $\lambda=1$. Recall that $t_{n} \rightarrow 0$ in $\tau(\mu)$ iff $\hat{\mu}\left(t_{n}\right) \rightarrow 1$.

If $\lambda<1,\left|\hat{\mu}\left(t_{n}\right)\right| \leq\left|\cos \left(2 \pi c_{n} t_{n}\right)\right|$. For $|\cos (2 \pi \lambda)|=1$ we must have $\cos (2 \pi \lambda)=$ \pm 1 , so that $2 \pi \lambda \in \mathbb{Z} \cdot \pi$. Since $0<\lambda<\frac{1}{2}, 2 \pi \lambda$ cannot be an integer multiple of $\pi$, so $|\cos (2 \pi \lambda)|<1$. Thus $t_{n}$ does not converge to 0 in $\tau(\mu)$.

If $\lambda=1$,

$$
\hat{\mu}\left(t_{n}\right)=\prod_{k=1}^{n} \cos \left(2 \pi 3^{n !-k !}\right) \cdot \prod_{k>n} \cos \left(2 \pi 3^{n !-k !}\right)=\prod_{k>n} \cos \left(2 \pi 3^{n !-k !}\right) .
$$

There exists $\omega>0$ so that for $0 \leq x \leq \omega, \cos (2 \pi x) \geq 1-49 x^{2}$. Hence

$$
\hat{\mu}\left(t_{n}\right) \geq \prod_{k>n}\left(1-49 \cdot 6^{n !-k !}\right)
$$

as long as $k>n$ and $n$ is such that $3^{n !-k !} \leq 3^{n !-(n+1) n !}=3^{-n \cdot n !}<\omega$.

Since for $a \in[0,1 / 2]$,

$$
\lim _{p \rightarrow \infty}\left(1-49 a^{p}\right)^{p}=1
$$

uniformly, and the function $p \mapsto\left(1-49 a^{p}\right)^{p}$ is increasing for any $a<1$, given $1>\delta>0$, there exists a $p=p(\delta)<+\infty$, so that

$$
\left(1-49 a^{p}\right)>(1-\delta)^{1 / p}
$$


for all $a \in[0,1 / 2]$. Hence letting $a=\frac{1}{6}$, we get that for any $n$ so that $k !-n ! \geq$ $n \cdot n !>p$,

Hence

$$
\hat{\mu}\left(t_{n}\right) \geq \prod_{k>n}(1-\delta)^{1 /(k !-n !)}
$$

$$
\log \hat{\mu}\left(t_{n}\right) \geq \sum_{k>n} \log (1-\delta) \frac{1}{k !-n !}
$$

Since $\log (1-\delta)<0$ and

$$
\sum_{k<n} \frac{1}{k !-n !} \leq \sum_{k<n} \frac{1}{k !-\frac{k !}{n}}=\sum_{k<n} \frac{1}{k !} \cdot \frac{1}{1-\frac{1}{n}} \leq \sum_{k<n} \frac{1}{k !}<e,
$$

we get that

$$
\log \hat{\mu}\left(t_{n}\right) \geq e \log (1-\delta)
$$

Hence

$$
\hat{\mu}\left(t_{n}\right) \geq(1-\delta)^{e}
$$

for any $n$ so that (1) $3^{-n \cdot n !}<\omega$ and $(2) n \cdot n !>p(\delta)$. It follows that $\hat{\mu}\left(t_{n}\right) \rightarrow 1$ as $n \rightarrow \infty$.

Now fix $0<\lambda<\frac{1}{2}$ and set

$$
\mu_{\lambda}(X)=\mu(\lambda \cdot X)
$$

for any Borel set $X \subset \mathbb{R}$. Then

$$
\hat{\mu}_{\lambda}(t)=\hat{\mu}(t / \lambda) .
$$

It follows that for any $0<\nu \leq \lambda$, the sequence $\nu 3^{n !}$ is convergent in $\tau\left(\mu_{\lambda}\right)$ iff $\nu=\lambda$. It follows that $\left\{\tau\left(\mu_{\lambda}\right): 0<\lambda<\frac{1}{2}\right\}$ are pairwise non-equivalent.

The author is indebted to U. Haagerup for communicating to him the following example:

Theorem 2.4. There exists a measure $\mu$, so that $\mu$ as well as $\mu * \cdots * \mu$ (any number of times) are singular with respect to Lebesgue measure, but $\tau(\mu)$ is the usual topology on the additive group of real numbers.

Proof. Let $\mu$ be as in the proof of Theorem 2.3 with $c_{n}=3^{-n}$. Then

$$
\hat{\mu}(t)=\prod_{k \geq 1} \cos \left(2 \pi \frac{t}{3^{k}}\right)
$$

We first claim that $\tau(\mu)$ is the usual topology on the real line. Assume that $t_{n} \rightarrow \infty$, but $\hat{\mu}\left(t_{n}\right) \rightarrow 1$. Choose $N$ so that for all $n>N, t_{n}>9$. Choose $k$ so that $c=t_{n} 3^{-k}>1$ but $t_{n} 3^{-k-1}=c / 3 \leq 1$. Then

$$
\begin{aligned}
\hat{\mu}(t) & \leq \cos \left(2 \pi t_{n} 3^{-k}\right) \cdot \cos \left(2 \pi t_{n} 3^{-k-1}\right) \cdot \cos \left(2 \pi t_{n} 3^{-k-2)}\right. \\
& =\cos (2 \pi c) \cdot \cos (2 \pi c / 3) \cdot \cos (2 \pi c / 9),
\end{aligned}
$$

where $1<c \leq 3$. Let

$$
f(c)=\cos (2 \pi c) \cdot \cos (2 \pi c / 3) \cdot \cos (2 \pi c / 9) .
$$

It is not hard to see that $f(c)$ is strictly less than 1 on the interval $1<c \leq 3$. It follows that $\hat{\mu}(t)<1$ whenever $t>9$. Contradiction.

All convolution powers of $\mu$ are singular with respect to Lebesgue measure (see the discussion of Taylor-Johnson measures [8] for examples of similar measures $\mu$ but satisfying even stronger properties than what we need here). 
2.3. Bimodule decompositions of crossed products. Let $(M, \phi)$ be a von Neumann algebra, $\phi$ a faithful normal state, and let $G$ be a locally compact abelian group. Assume that $\alpha$ is an action of $G$ on $M$, which leaves $\phi$ invariant. Then the crossed product von Neumann algebra $C=M \rtimes_{\alpha} G$ contains a canonical copy $A$ of the group algebra $L(G) \cong L^{\infty}(\hat{G})$; moreover, the state $\phi$ gives rise to a normal faithful conditional expectation $E: C \rightarrow A$. Let $\hat{\psi}$ be a normal faithful weight on $A$, and let $\psi=\hat{\psi} \circ E$. This is a normal faithful weight on $C$. Moreover, $L^{2}(C, \psi)$ is an $A, A$-bimodule in a natural way.

Fix an isomorphism $(A, \hat{\psi}) \cong L^{\infty}\left(\hat{G}, \nu_{G}\right)$. Denote by $\ell^{2}$ the Hilbert space with basis $e_{1}, e_{2}, \ldots$ and by $\ell_{n} \subset \ell^{2}$ the subspaces spanned by $e_{1}, \ldots, e_{n}$. Given a measure $\eta$ on $X \times X$ whose projections onto the the coordinate directions on $X \times X$ are both equivalent to $\nu_{G}$, and a multiplicity function $n: X \times X \rightarrow \mathbb{N} \cup\{\infty\}$, let

$$
H(\eta, n)=L^{2}(X \times X, \eta, n)
$$

be the space of square-integrable functions from $X \times X \rightarrow \ell^{2}$, so that $f(x, y) \in$ $\ell_{n(x, y)}$ for all $x, y \in X$ (where for convenience we set $\ell_{\infty}^{2}=\ell^{2}$ ). Endow $H(\eta, n)$ with an $A, A$-bimodule structure by letting

$$
(f \cdot h \cdot g)(x, y)=f(x) h(x, y) g(y), \quad f, g \in A, \quad h \in H .
$$

In fact [2, 11, 5] any bimodule over $A$ can be represented in this way. It is easily seen that if $\eta^{\prime}$ is another measure on $X \times X$, projecting onto $\mu$, and $\eta^{\prime}$ is mutually absolutely continuous with $\eta$, then $H(\eta, n) \cong H\left(\eta^{\prime}, n\right)$ as bimodules over $A$.

Choose vectors $\xi_{1}, \xi_{2}, \ldots \in L^{2}(M, \phi),\left\|\xi_{1}\right\|_{2}=1,\left\|\xi_{i}\right\|_{2} \leq 1$, a measure $\mu$ on $\hat{G}$ and a multiplicity function $n: \hat{G} \rightarrow \mathbb{N}$, so that

$$
\begin{aligned}
\alpha_{g}\left(\xi_{i}\right) \perp \alpha_{h}\left(\xi_{j}\right) & \forall i, j \quad \forall g, h \in G, \\
L^{2}(M, \phi)= & \overline{\operatorname{span}}\left\{\alpha_{g}\left(\xi_{i}\right): g \in G, i=1,2, \ldots\right\}, \\
\left\langle\xi_{i}, \alpha_{g}\left(\xi_{j}\right)\right\rangle= & \hat{\mu}_{i}(g),
\end{aligned}
$$

where $\mu_{i}=\left.\mu\right|_{n^{-1}(i)}$ and $\hat{\imath}$ denotes the Fourier transform. Let $(X, \mu)=\left(\hat{G}, \nu_{G}\right)$, where $\nu_{G}$ is the Haar measure on $G$. Let

$$
\eta(x, y)=\mu(x-y), \quad n(x, y)=n(x-y)
$$

be a measure and a multiplicity function on $\hat{G} \times \hat{G}$, and let $H=H(\eta, n)$ (note that the projections of $\eta$ onto the coordinate directions are precisely $\left.\mu * \nu_{G}=\mu(\hat{G}) \cdot \nu_{G}\right)$. We claim that $H(\eta, n) \cong L^{2}(C, \psi)$ as bimodules. To see this, one can verify that the map $p \xi_{i} p \mapsto p(x) p(y) \chi_{n^{-1}(\{i\})}$ for a projection $p \in L^{\infty}(\hat{G})$ with $\hat{\psi}(p)<+\infty$ is a bimodule isometry from the linear span of $p \xi_{i} p \in L^{2}(C)$ to $H(\eta, \mu)$.

Note that the measure $\mu$ (which is the "spectral measure" of the representation of $G$ on $\left.L^{2}(M)\right)$ is uniquely determined up to absolute continuity.

\section{Full type III FACTORS}

Assume that $M$ is a full factor, so that its group of inner automorphisms $\operatorname{Inn}(M)$ is a closed subgroup of the group of all automorphisms $\operatorname{Aut}(M)$, endowed with the $u$-topology [9, 4]. Let $\operatorname{Out}(M)=\operatorname{Aut}(M) / \operatorname{Inn}(M)$, with the quotient topology. Denote by $\pi$ the quotient map from $\operatorname{Aut}(M)$ to $\operatorname{Out}(M)$, and by $\delta$ the composition $\pi \circ \sigma_{t}^{\phi}$ (which is independent of $t$ by Connes' Radon-Nikodym type theorem [3]).

Assume that the action of $\mathbb{R}$ on $M$ by $t \mapsto \sigma_{t}^{\phi}$ extends, for some $\phi$, to an action of a locally compact completion $G$ of $\mathbb{R}$ (by Proposition 2.1 above, this means that 
either $G$ is just $\mathbb{R}$, or $G$ is compact, and $\phi$ is almost-periodic). In this case, call $\phi$ a $G$-state (or weight) on $M$. Call the crossed product

$$
M \rtimes_{\sigma^{\phi}} G
$$

the $G$-core of $M$. It is known [3, 4] that the $G$-core of $M$ is independent of the choice of the state $\phi$ (having the property that its modular group extends to $G$ ).

Definition 3.1. Let $M$ be full. Then define:

(a) $\tau(M)$ to be the weakest topology on $\mathbb{R}$ making the map

$$
\delta: \mathbb{R} \rightarrow \operatorname{Out}(M)
$$

continuous (this invariant was introduced by Connes in [4]).

(b) The $\mathscr{S}$ invariant to be the intersection

$$
\mathscr{S}(M)=\bigcap_{\phi \text { f.n.state on } M} \mathscr{C}_{\oplus_{n}\left(\Delta^{\phi}\right)^{\otimes n} .}
$$

Part (b) of the definition is equivalent to the Sd invariant of Connes [4] if the intersection were to be taken over all faithful normal almost-periodic weights.

Note that since we are dealing only with states in the definition of $\mathscr{S}(M)$, the delta measure at 1 is always in $\mathscr{S}(M)$.

Assume that the $G$-core of $M$ is a factor. Note that since the $G$-core has a semifinite normal trace, it is full iff its compression by a finite projection is non$\Gamma$. In particular, if the $G$ core is a factor and is full, it has no non-trivial central sequences.

Theorem 3.2. Assume that for some $G$-state $\phi$ on a factor $M$ the $G$-core is a full factor. Then $M$ is full.

Proof. Let $C=M \rtimes_{\sigma} G$ be the $G$-core of $M$. Assume for contradiction that $M$ is not full. Then by [4, Corollary 3.6, Proposition 2.8] there exists a sequence of unitaries $m_{n} \in M,\left|m-\phi\left(m_{n}\right)\right| \nrightarrow 0$, and so that $\left[m_{n}, m\right] \rightarrow 0 *$-strongly for all $m \in M$ and $\left[m_{n}, \phi\right] \rightarrow 0$ in norm on $M_{*}$ for any $\phi \in M_{*}$. View $m_{n} \in C \supset M$. We claim that $\left\{m_{n}\right\}$ is a non-trivial central sequence in $C$. Let $E: C \rightarrow L(G) \subset C$ be the canonical conditional expectation. Then $E\left(m_{n}\right)=\phi\left(m_{n}\right)$, thus $E\left(m_{n}\right)-m_{n}$ does not go to zero $*$-strongly, so that $m_{n}$ is not asymptotically scalar. For any $m \in M,\left[m_{n}, m\right] \rightarrow$ $0 *$-strongly. Denote by $U_{g} \in L(G) \subset C, g \in G$, the implementing unitaries. Then by Connes' results [4, Theorem 2.9(3)], for any $t \in \mathbb{R} \subset G,\left[m_{n}, U_{g}\right] \rightarrow 0 *$-strongly. Hence $\left[m_{n}, u\right] \rightarrow 0 *$-strongly for any $u \in W^{*}\left(M, U_{t}: t \in \mathbb{R}\right)=C$. Hence $m_{n}$ is an asymptotically central sequence in $C$. Since $C$ is assumed to be a full factor, we have arrived at a contradiction.

Theorem 3.3. Assume that for some $G$-state $\phi$ on $M$, the $G$-core of $M$ is a full factor. Then if for some $H$ not necessarily locally compact containing $\mathbb{R}$ as a dense subgroup, there is an $H$-weight $\psi$ on $M$, one must have that $H \subset G$.

Proof. Let $C=M \rtimes_{\sigma^{\phi}} G$. Let $L(G) \subset C$ be the canonical copy of the group algebra of $G$; for $g \in G$, denote by $w_{g} \in L(G)$ the implementing unitary. We write $E_{L(G)}$ for the canonical conditional expectation from $C$ onto $L(G)$.

Assume that $H \not \subset G$, so that the topology defined by the inclusion $\mathbb{R} \subset H$ is not stronger than the topology defined by the inclusion $\mathbb{R} \subset G$. Hence there 
exists a sequence $t_{n} \in \mathbb{R}$, so that $\sigma_{t_{n}}^{\psi} \rightarrow \mathrm{id}$, but $\sigma_{t_{n}}^{\phi}$ does not converge. Let $u_{t}=[\phi: \psi]_{t} \in M \subset C$. It follows that

$$
\left.\operatorname{Ad}_{u_{t} w_{t}}\right|_{C}=\sigma_{t}^{\psi}
$$

In particular, $\left[u_{t_{n}} w_{t_{n}}, x\right] \rightarrow 0 *$-strongly for all $x \in M \subset C$. Note moreover that $u_{t} w_{t}$ commutes with $u_{s} w_{s}$ (since they form a one-parameter subgroup of $U(C)$ ). Hence for $s$ fixed, $\left[u_{t_{n}} w_{t_{n}}, u_{s} w_{s}\right]=0$, and since $u_{t_{n}} w_{t_{n}}$ asymptotically commutes with $M \subset C$, it also follows that $\left[u_{t_{n}} w_{t_{n}}, w_{s}\right] \rightarrow 0 *$-strongly. It follows that $u_{t_{n}} w_{t_{n}}$ is a central sequence in $M$. Hence, by the assumption that $C$ is a full factor, and by the fact that $C$ is type $\mathrm{II}_{\infty}$, we find that $\lambda_{n} u_{t_{n}} w_{t_{n}} \rightarrow 1 *$-strongly for some scalars $\lambda_{n} \in \mathbb{T}$. But then

$$
E_{L(G)}\left(\lambda_{n} u_{t_{n}} w_{t_{n}}\right) \rightarrow 1
$$

*-strongly. Since $u_{t_{n}} \in M \subset C, E_{L(G)}\left(u_{t_{n}}\right) \in \mathbb{C}$, so that $\lambda_{n}^{\prime} w_{t_{n}} \rightarrow 1 *$-strongly for some sequence $\lambda_{n}^{\prime} \in \mathbb{T}$. Hence $\lambda_{n} \pi\left(t_{n}\right) \rightarrow 1 *$-strongly, where $\pi$ is the left regular representation of $G$ (since the representation of $G$ on $L^{2}(C, \operatorname{Tr}$ ) is a multiple of its left regular representation).

Now choose $\phi$ a function on $G$, supported in a compact neighborhood of identity and so that $\|\phi\|_{2}=1$. Then $\lambda_{n} \pi\left(t_{n}\right) \cdot \phi \rightarrow \phi$ in $L^{2}$. In particular, it means that the support $X$ of $\phi$ and its translate $X+t_{n}$ cannot be disjoint once $n$ is sufficiently large. It follows that $t_{n} \in X-X$ for sufficiently large $n$. It follows that $t_{n} \rightarrow 0$ in G. Contradiction.

Corollary 3.4. If the $G$-core of $M$ is full, then $\tau(M)=\tau_{G}$, the weakest topology making the inclusion $\mathbb{R} \subset G$ continuous.

We also have:

Proposition 3.5. If $M$ has a $G$-state and $H$ is a discrete subgroup, then the $H$-core of $M$ is not a full factor.

Proof. Let $\phi$ be a $G$-state on $M$, and denote by $C$ the $H$-core $M \rtimes_{\sigma^{\phi}} H$. Assume that $C$ is a factor. By assumption, there exists a sequence $t_{n} \in \mathbb{R}, t_{n} \rightarrow 0$ in the topology of $G$, but $t_{n}$ not convergent in $H$. Denote by $w_{h} \in C, h \in H$, the implementing unitaries for the $H$ action on $M$. Then $\operatorname{Ad}_{w_{t_{n}}}(x) \rightarrow x$ *-strongly for all $x \in M \subset C$; moreover, $\operatorname{Ad}_{w_{t_{n}}}(w)=w$ for all $w \in W^{*}\left(w_{h}: h \in H\right)=L(H)$. Hence $w_{t_{n}}$ form a central sequence. Arguing exactly as in the last paragraph of the proof of Theorem 3.3 we find that for no sequence of scalars $\lambda_{n} \in \mathbb{T}$ does $\lambda_{n} w_{t_{n}} \rightarrow 1$ *-strongly in the group algebra $L(H)$. Hence $w_{t_{n}}$ is a non-trivial central sequence in $M$.

Choose $p \in L(H) \subset C$ a projection of finite trace. Then $\left[p, w_{t_{n}}\right]=0$ and hence $p w_{t_{n}} p$ is a central sequence in $p C p$, which has a finite trace. Thus $p C p$ has property $\Gamma$. Hence by Connes' results [4], $p C p$ and hence $C$ is not full.

\section{Crossed products, Free entropy dimension And the $\mathscr{S}$ invariant}

The main result of this section is a computation of the $\mathscr{S}$ invariant of some type III factors $M$, for which the core has a sequence of generators with large free entropy dimension. We first recall some preliminaries. 
4.1. Free entropy dimension for infinite families of generators. It is useful for us to consider Voiculescu's free entropy dimension in the context of an infinite family of generators $x_{1}, x_{2}, \ldots$ in a von Neumann algebra $M$. We point out the necessary modifications of Voiculescu's approach ([20], [21]; see also [18], where a similar modification was necessary). We freely use the notations of [19], 20], [21].

Let $x_{i}, 1 \leq i<+\infty$ and $y_{i}, 1 \leq i<+\infty$ be in $M$. Fix a free ultrafilter $\omega$ and an element $\kappa$ in the Stone-Chech compactification of $(0,1]$, not lying in this interval. Define

$$
\chi^{\omega}\left(x_{1}, \ldots, x_{p}: y_{1}, y_{2}, \ldots ; m, \epsilon\right)=\liminf _{q \rightarrow \infty} \chi^{\omega}\left(x_{1}, \ldots, x_{p}: y_{1}, \ldots, y_{q} ; m, \epsilon\right)
$$

(note that the liminf is actually a limit in this definition).

Define $\chi^{\omega}\left(x_{1}, \ldots, x_{p}: y_{1}, y_{2}, \ldots\right)$ in exactly the same way as in [20], but using $\chi$ defined above.

One still has the property

$$
\chi^{\omega}\left(x_{1}, \ldots, x_{p}: y_{1}, y_{2}, \ldots\right) \leq \chi^{\omega}\left(x_{1}, \ldots, x_{p}: z_{1}, \ldots, z_{l}\right)
$$

for all $z_{1}, \ldots, z_{l} \in W^{*}\left(x_{1}, \ldots, x_{p}, y_{1}, y_{2}, \ldots\right)$.

Let $S_{1}, \ldots, S_{p}$ be a free semicircular family, free from $\left\{x_{1}, \ldots, x_{p}\right\} \cup\left\{y_{1}, y_{2}, \ldots\right\}$. Set $x_{j}^{\varepsilon}=x_{j}+\varepsilon S_{j}$. Then define

$$
\begin{aligned}
& \delta_{\omega, \kappa}^{0}\left(x_{1}, x_{2}, \ldots, x_{p}: y_{1}, y_{2}, \ldots\right) \\
& \quad=p-\lim _{\epsilon \rightarrow \kappa} \frac{\chi^{\omega}\left(x_{1}^{\varepsilon}, \ldots, x_{p}^{\varepsilon}: S_{1}, \ldots, S_{p}, y_{1}, y_{2}, \ldots\right)}{\log \epsilon} .
\end{aligned}
$$

Now define

$$
\underline{\delta}\left(x_{1}, x_{2}, \ldots\right)=\liminf _{p \rightarrow \infty} \delta_{\omega, \kappa}^{0}\left(x_{1}, \ldots, x_{p}: x_{p+1}, x_{p+2}, \ldots\right) .
$$

For a finite family $x_{1}, \ldots, x_{n}$ this is exactly Voiculescu's definition of free entropy dimension. In general,

$$
\underline{\delta}\left(x_{1}, x_{2}, \ldots\right) \leq \liminf _{p \rightarrow \infty} \delta_{\omega, \kappa}^{0}\left(x_{1}, \ldots, x_{p}\right) .
$$

Moreover,

$$
0 \leq \underline{\delta}\left(x_{1}, x_{2}, \ldots\right)
$$

iff $W^{*}\left(x_{1}, x_{2}, \ldots\right)$ can be embedded into $R^{\omega}$, the ultrapower of the hyperfinite $\mathrm{II}_{1}$ factor.

If $x_{1}, \ldots, x_{p}$ are free form $x_{p+1}, x_{p+2}, \ldots$, then

$$
\delta_{\omega, k}^{0}\left(x_{1}, \ldots, x_{p}: x_{p+1}, \ldots\right)=\delta_{\omega, \kappa}^{0}\left(x_{1}, \ldots, x_{p}\right) .
$$

In particular, if the families $\left\{x_{1}\right\}, \ldots,\left\{x_{p}\right\}, \ldots,\left\{y_{1}, y_{2}, \ldots\right\}$ are free and $\left\{y_{1}, y_{2}, \ldots\right\}^{\prime \prime}$ is embeddable, we get by [21] that

$$
\begin{aligned}
\underline{\delta}\left(x_{1}, y_{1}, x_{2}, y_{2}, \ldots\right) & =\lim _{p \rightarrow \infty} \delta_{\omega, k}^{0}\left(x_{1}, \ldots x_{p}, y_{1}, \ldots, y_{p}: y_{1}, y_{2}, \ldots\right) \\
& =\lim _{p \rightarrow \infty} \delta_{\omega, k}^{0}\left(x_{1}, \ldots, x_{p}\right)+\underline{\delta}\left(y_{1}, y_{2}, \ldots\right) \\
& \geq \sum_{k} \delta\left(x_{k}\right) .
\end{aligned}
$$


Definition 4.1. Let $M$ be a $\mathrm{II}_{1}$ von Neumann algebra. Denote by

$$
\delta(M)=\sup _{x_{1}, x_{2}, \ldots \in M} \underline{\delta}\left(x_{1}, x_{2}, \ldots\right),
$$

where the supremum is taken over all self-adjoint families (finite or infinite) $x_{1}$, $x_{2}, \ldots$ of generators of $M$. If $N$ is type $\mathrm{II}_{\infty}$, we write $\delta(N)$ for the supremum over all finite-trace projections $p \in N$ of $\delta(p N p)$.

Remark 4.2. It is quite likely that $\delta(M) \in\{0,1,+\infty\}$ if $M$ is type $\mathrm{II}_{\infty}$. Note also that $\delta(M) \leq \delta(M \otimes B(H))$ for all $M$.

While $\delta(M)$ is clearly an invariant of $M$, our inability to prove that the number $\delta\left(x_{1}, x_{2}, \ldots\right)$ is independent of the choice of generators $x_{1}, x_{2}, \ldots, 19,20,21$. results in the inability to compute the exact value of $\delta$ for infinite-dimensional von Neumann algebras. However, as we pointed out above, if $M=L\left(\mathbb{F}_{n}\right) * N$, with $N \subset R^{\omega}$ and $n=1,2, \ldots$ or $+\infty$, we have $\delta(M) \geq n$ and $\delta(M \otimes B(H)) \geq n$ (in fact, $=+\infty$ by [7]). Furthermore, as Voiculescu proved in [20], $\delta(R)=1$ if $R$ is the hyperfinite $\mathrm{II}_{1}$ (or of $\mathrm{II}_{\infty}$ ) factor; more generally, $\delta(M) \leq 1$ if $M$ has property $\Gamma$ or has a Cartan subalgebra (since these properties are inherited by compressions of a von Neumann algebra, these statements are valid for $M$ of type $\mathrm{II}_{1}$ or of type $\mathrm{II}_{\infty}$ ). It is also known that $\delta(M)>1$ implies that the center of $M$ is at most atomic.

The following theorem essentially follows from the results of [20]; we sketch the necessary modifications of the proof coming from the fact that we may be dealing with infinite families of generators.

Theorem 4.3. Let $M$ be a $I I_{1}$ or $I I_{\infty}$ factor. Let $L^{\infty}(X, \mu) \cong A \subset M$ be a diffuse abelian subalgebra, so that $\left.\operatorname{Tr}_{M}\right|_{A}$ is semifinite. View $L^{2}(M)$ as an $A, A$ bimodule, and identify it with $H(\eta, n)$ for some measure $\eta$ on $X \times X$. Assume that $\eta$ is disjoint from $\mu \times \mu$, i.e., $X \times X=Y_{1} \cup Y_{2}$, so that $\eta\left(Y_{1}\right)=0$ and $(\mu \times \mu) Y_{2}=0$. Then $\delta(M) \leq 1$.

Proof. We first reduce to the case that $M$ is type $\mathrm{II}_{1}$. Given $t \in(0,+\infty)$, let $p \in A$ be a finite projection, corresponding to the characteristic function of some set $Y \subset X, \mu(Y)<+\infty$. Then view $p M p$ as a bimodule over $p A p$. It is not hard to see that $p M p$ can be identified with $H\left(\eta^{\prime}, n^{\prime}\right)$, with $\eta^{\prime}$ absolutely continuous with respect to $\left.\eta\right|_{Y \times Y \subset X \times X}, n^{\prime}=\left.n\right|_{Y \times Y \subset X \times X}$. If $\eta$ is disjoint from $\mu \times \mu$, then $\eta^{\prime}$ is disjoint from $\mu^{\prime}=\left.\mu\right|_{Y}$. If the statement of the theorem can be proved for $p M p$ and $p A p \subset p M p$, we would have that $\delta(q M q) \leq 1$ for all $q \in M$ of finite trace (since $q M q$ depends up to isomorphism only on the center-valued trace of $q$ ). Hence by definition we get that $\delta(M) \leq 1$.

Let $x_{1}, x_{2}, \ldots \in M$ be a sequence of generators of $M$; by rescaling (which does not affect $\left.\underline{\delta}\left(x_{1}, x_{2}, \ldots\right)\right)$, assume that $\left\|x_{j}\right\|=1$. By the hypothesis, given $\omega, \delta>0$ and a measure $\eta^{\prime}$ in the absolute continuity class of $\eta$, there exists an $N=N\left(\eta^{\prime}, \omega, \delta\right)$ and a finite family of $N$ disjoint measurable subsets $X_{i}, i \in I$ of $X$, each of measure $1 / N$, a subset $K \subset I \times I$, so that $X=\bigcup X_{i}$ and $\eta^{\prime}\left(Y_{2} \backslash \bigcup_{(i, j) \in K} X_{i} \times X_{j}\right)<\omega$, $(\mu \times \mu)\left(\bigcup_{(i, j) \in K} X_{i} \times X_{j}\right)=|K| / N^{2}<\delta$. It follows that for each fixed $T, \delta$ and $\omega$, there are projections $p_{1}, \ldots, p_{N} \in A$ of trace $1 / N$ (corresponding to the characteristic functions of $X_{1}, \ldots, X_{N}$ in the identification $\left.A \cong L^{\infty}(X, \mu)\right)$, so that

$$
\left\|x_{t}-\sum_{i, j \in K} p_{i} x_{t} p_{j}\right\|_{2}<\omega
$$


and

$$
\frac{|K|}{N^{2}}<\delta
$$

for all $1 \leq t \leq T$. Using Voiculescu's result 20 and the fact that $p_{1}, \ldots, p_{n} \in$ $W^{*}\left(x_{1}+\sqrt{\epsilon} S_{1}, \ldots, x_{T}+\sqrt{\epsilon} S_{T}, S_{1}, \ldots, S_{T}, x_{T+1}, x_{T+2}, \ldots\right)$, we get the estimate

$$
\begin{array}{r}
\chi\left(x_{1}+\sqrt{\epsilon} S_{1}, \ldots, x_{T}+\sqrt{\epsilon} S_{T}: S_{1}, \ldots, S_{T}, x_{T+1}, x_{T+2}, \ldots\right) \\
\leq \chi\left(x_{1}+\sqrt{\epsilon} S_{1}, \ldots, x_{T}+\sqrt{\epsilon} S_{T}: p_{1}, \ldots, p_{N}\right) \\
\leq(T(1-\delta)-1) \log (\epsilon+\omega)+C,
\end{array}
$$

where $C$ is a constant, independent of $\omega, \epsilon$ and $\delta$. Letting $\omega \rightarrow 0$ first, we conclude that

$$
\begin{array}{r}
T-\lim _{\epsilon \rightarrow \kappa} \frac{\chi\left(x_{1}+\sqrt{\epsilon} S_{1}, \ldots, x_{T}+\sqrt{\epsilon} S_{T}: S_{1}, \ldots, S_{T}, x_{T+1}, x_{T+2}, \ldots\right)}{\log \epsilon} \\
\leq T-T(1-\delta)+1=1+\delta T .
\end{array}
$$

Since $\delta>0$ is arbitrary, it follows that

$$
\delta_{\omega, \delta}^{0}\left(x_{1}, \ldots, x_{T}: x_{T+1}, x_{T+2}, \ldots\right) \leq 1
$$

for all $T$. Hence $\underline{\delta}\left(x_{1}, x_{2}, \ldots\right) \leq 1$. Since the sequence of generators $\left\{x_{j}\right\}$ was arbitrary, we get that $\delta(M) \leq 1$.

In a similar way one sees that $\delta(M)>1$ implies that $M$ is a non- $\Gamma$ factor.

4.2. Free entropy dimension and crossed products. Using the estimates in [20], stated in Theorem 4.3) we record the following theorem (due to Voiculescu, but formulated by him under the additional hypothesis that $M$ be finitely generated):

Theorem 4.4. Let $(M, \phi)$ be a von Neumann algebra. Let $\alpha$ be an action of a locally compact non-discrete abelian group $G$ on $M$. Assume that $\alpha$ preserves the state $\phi$ on $M$. Denote by $U_{g}: L^{2}(M, \phi) \rightarrow L^{2}(M, \phi)$ the unitaries extending $\alpha(g): M \rightarrow M$. Let $\mu \in M(\hat{G})$ be the spectral measure of the representation $g \mapsto \bigoplus_{n}(U \oplus \bar{U})_{g}^{\otimes n}$ (here $\bar{U}$ denotes the conjugate representation). Let $C=\left(M \rtimes_{\alpha} G\right) \otimes B(H)$. Assume that for some normal faithful weight $\psi$ on $L(G)$, the composition $\psi \circ E_{L(G)}: C \rightarrow \mathbb{R}$ is a normal faithful semifinite trace on $C$.

Then if $C$ is a factor and satisfies $\delta(C)>1, \mathscr{C}_{\mu}$ must contain the Haar measure of $G$.

Proof. View $C$ as a bimodule over the abelian subalgebra $L(G, \gamma) \cong L^{\infty}(G, \mu)$ of $M \rtimes_{\alpha} G$. This bimodule can be identified with $H(\eta, n)$ for some measure $\eta$ on $\hat{G} \times \hat{G}$ and some multiplicity function $n$ (see $\$ 2.3$.

By Theorem 4.3. $\eta$ cannot be disjoint from the product measure $\nu_{\hat{G}} \times \nu_{\hat{G}}$ on $\hat{G} \times \hat{G}$. It follows that $\mu$ cannot be disjoint from the Haar measure $\nu_{\hat{G}}$. We may assume that $\mu$ is symmetric. We claim that $\nu_{\hat{G}}$ is absolutely continuous with respect to $\mu^{\prime}=\sum_{n \geq 1} \frac{1}{2^{n}} \mu^{* n}$, which is the spectral measure of $g \mapsto \bigoplus_{n} U_{g}^{\otimes n}$. We must show that if $\mu^{\prime}(X)=0$, then also $\nu_{\hat{G}}(X)=0$ for all Borel subsets $X \subset \hat{G}$. Assume to the contrary that $\nu_{\hat{G}}(X)>0$ but $\mu^{\prime}(X)=0$. Then $\mu^{* n}(X)=0$ for all $n$. Since $\mu$ is not disjoint from $\nu_{G}$, there exists a subset $Y$ of $\hat{G}$, for which $\left.\nu_{\hat{G}}\right|_{Y}=\left.f \cdot \mu\right|_{Y}$. By modifying $\mu$ and $Y$, we may assume that $f=1$ and $\mu(Y)=\nu_{\hat{G}}(Y)=1$. Then $\nu_{\hat{G}}=f_{n} \cdot \mu^{* n}$ on $n Y=Y+Y+\ldots+Y$ ( $n$ times $)$. Moreover, since $\mu$ and $\nu_{\hat{G}}$ are 
symmetric, we may assume that $Y=-Y$. Hence we find that $\nu_{\hat{G}}=f \cdot \mu^{\prime}$ on the subgroup of $\hat{G}$ generated by $Y$.

Clearly, $X$ (modulo a set of $\nu_{G}$-measure zero) is contained in the complement of $H$, and $\nu_{G}(H) \neq 0$. Since $\nu_{G}$ is $\sigma$-finite, there exists a countable sequence $x_{n} \in \hat{G}$, so that (after possibly making $X$ smaller by a set of $\nu_{G}$-measure zero) $X \subset \bigcup_{n}\left(x_{n}+H\right)$.

Let $x \in \hat{G}$. Then for any measure $\sigma$ on $\hat{G}$ of finite total mass,

$$
\left(\left.\mu_{a} * \nu_{G}\right|_{H}\right)(x)=\sigma(x+H) \text {. }
$$

It follows that if there are finite measures $\sigma_{n}$, absolutely continuous with respect to $\mu^{\prime}$ and so that $\sigma_{n}\left(x_{n}+H\right) \neq 0$, then $\mu^{\prime} *\left(\sum \frac{1}{2^{n}} \sigma_{n}\right)$ (and hence $\mu^{\prime} * \mu^{\prime}$ and hence $\mu^{\prime} \sim \mu^{\prime} * \mu^{\prime}$ ) gives $X$ a non-zero measure (which would be a contradiction). Hence $\mu^{\prime}\left(x_{n}+H\right)=0$ for some $n$. Let $p \in L^{\infty}(\hat{G})$ be the projection corresponding to the characteristic function of $H$, and let $q \in L^{\infty}(\hat{G})$ be the projection corresponding to the characteristic function of $H+x_{n}$. Let $(s, t) \in H \times\left(H+x_{n}\right) \subset \hat{G} \times \hat{G}$. Then $s-t \in H-H+x_{n} \in H+x_{n}$. Since $\mu^{\prime}\left(x_{n}+H\right)=0$, it follows that the characteristic function of $H \times\left(H+x_{n}\right)$ is zero in $L^{2}(\hat{G} \times \hat{G}, \eta, n)$, where $\eta$ and $n$ are as in 82.3 But this implies that $p C q=0$, so that $p$ and $q$ are not equivalent in $C$. Hence $C$ is not a factor. Contradiction.

It should be noted that this theorem is of interest even in the type $\mathrm{II}_{\infty}$ case. For example, we get as a consequence that an amplification of a free group factor $L\left(\mathbb{F}_{n}\right) \otimes B(H)$ cannot be written as a crossed product $N \rtimes_{\alpha} G$, with $G$ abelian, unless the spectral measure of $\alpha$ contains Lebesgue measure on $G$.

\subsection{Consequences for the $\mathscr{S}$ invariant.}

Corollary 4.5. Assume that for some normal faithful $G$ state $\phi$ on $M$, the $G$ core $C$ of $M$ satisfies $\delta(C)>1$. Then for any other n.f.s. weight $\psi$ on $M$, the Haar measure on $\hat{G}$ is contained in the spectral measure of the action of $G$ on $\bigoplus_{n} L^{2}(M, \psi)^{\otimes n}$.

Proof. This is immediate from $C=M \rtimes_{\sigma^{\phi}} G$ and Theorem 4.4.

Theorem 4.6. Assume that the core $C$ of $M$ satisfies $\delta(C)>1$. Assume that there exists a state $\phi$ on $M$, for which the spectral measure of the modular group is $\lambda+\delta_{1}$. Then $\mathscr{S}(M)=\mathscr{C}_{\lambda+\delta_{1}}$.

Proof. By Corollary 4.5 we get that $\mathscr{C}_{\lambda} \subset \mathscr{S}(M)$. Because $\phi$ is a state, $\mathscr{S}(M) \subset$ $\mathscr{C}_{\lambda+\delta_{1}}$.

\section{Applications to free Araki-Woods factors}

5.1. $G$-core for certain free Araki-Woods factors. Let $\hat{G} \subset \mathbb{R}$, and denote by $\sigma$ its Haar measure.

Let $\nu$ be a measure on $\hat{G}$, which is symmetric, $\nu(X)=\nu(-X)$. Extending $\nu$ to all of $\mathbb{R}$ by $\nu(X)=\nu(X \cap \hat{G})$ gives us a measure on the real line.

Let $H=L^{2}(\mathbb{R}, \nu)=L^{2}(\hat{G}, \nu)$. Denote by $H_{\mathbb{R}}$ the subspace of $H$ consisting of functions with the property that $f(x)=\overline{f(-x)}$. Then $H_{\mathbb{R}}$ is a real subspace of $H$, and the restriction of the inner product on $H$ to $H_{\mathbb{R}}$ is real-valued. Moreover, the one-parameter group of unitary operators

$$
U_{t}: t \mapsto \mathscr{M}_{\exp (i t)}
$$


of multiplication operators acting on $H$ leaves $H_{\mathbb{R}}$ invariant and hence defines an action of $\mathbb{R}$ on this real Hilbert space.

Note that if we consider the dual inclusion $\mathbb{R} \subset G$, then the map

$$
t \mapsto \mathscr{M}_{\exp (i t)}: L^{2}(\hat{G}, \nu) \rightarrow L^{2}(\hat{G}, \nu)
$$

extends to the map

$$
g \mapsto \mathscr{M}_{\langle g, \cdot\rangle}: L^{2}(\hat{G}, \nu) \rightarrow L^{2}(\hat{G}, \nu),
$$

where $\langle g, \cdot\rangle$ denotes the function $\langle g, \cdot\rangle(\chi)=\chi(g), g \in G, \chi \in \hat{G}$. Hence $U_{t}$ extends to an action $U_{g}$ of $G$ on $H$; it is not hard to see that once again $H_{\mathbb{R}}$ is invariant under $U_{g}, g \in G$, and hence $G$ acts on the real Hilbert space $H_{\mathbb{R}}$ as well. Note that $U_{g}$ is isomorphic to the left regular representation of $G$. In particular, the spectral measure of the infinitesimal generator of $t \mapsto U_{t}$ is $\nu$.

Let $\Gamma\left(H_{\mathbb{R}}, U_{t}\right)^{\prime \prime}$ be the free Araki-Woods factor [15] associated to the action $U_{t}$ of $\mathbb{R}$ on $H_{\mathbb{R}}$, and let $\phi$ denote the free quasi-free state on $\Gamma\left(H_{\mathbb{R}}, U_{t}\right)^{\prime \prime}$. For convenience we shall write $\Gamma(\mu)$ for this von Neumann algebra.

Theorem 5.1. Let $\sigma$ be the Haar measure on $G$. Then the $G$-core of $M=\Gamma(\sigma)^{\prime \prime}$ is isomorphic to $L\left(\mathbb{F}_{\infty}\right) \otimes B(H)$.

Proof. We first note that in the case that $G$ is compact, the $G$-core is the so-called discrete core of $M$, and the claimed isomorphism was already proved by Dykema (6]; see [15] for the argument reducing the case of a general Araki-Woods factor to the form which can utilize Dykema's results). Therefore, we proceed under the assumption that $G$ is not compact and hence $G=\mathbb{R}, \nu_{\hat{G}}$ is the Lebesgue measure. In particular, $\nu_{\hat{G}}$ is non-atomic.

Let $C$ denote the core. Let $\xi \in H_{\mathbb{R}}$ be a cyclic vector for $U_{g}, g \in G$ (one can take, for example, any function $f(x)=f(-x)$, which is nowhere zero on $\hat{G}$, and which lies in $\left.L^{2}(\hat{G}, \sigma)\right)$. Let $\phi$ be the positive-definite function on $G$ associated to $\xi, \phi(g)=\left\langle\xi, U_{g} \xi\right\rangle$. Let $\mu$ be the Fourier transform of $\phi$ (viewed as a measure on $\hat{G})$. By [16] and [17],

$$
C \cong \Phi(L(G), \eta)
$$

where $\eta$ is a completely positive map from $L(G) \cong L^{\infty}(\hat{G}) \rightarrow L^{\infty}(\hat{G})$ given by

$$
h \mapsto h * \mu,
$$

* denoting the convolution on measures on $\hat{G}$. Notice that the measure $\mu$ is just the measure resulting from applying the state $\langle\xi, \cdot \xi\rangle$ to the spectral measure of the infinitesimal generator of $U_{g}$. Hence $\mu$ is absolutely continuous with respect to the Haar measure $\sigma$ on $\hat{G}$.

The $L(G), L(G)$ bimodule associated to this completely positive map is

$$
L^{2}(\hat{G} \times \hat{G}, \hat{\mu}),
$$

where $\hat{\mu}\left(\chi, \chi^{\prime}\right)=\mu\left(\chi-\chi^{\prime}\right)$, with $L(G) \cong L^{\infty}(\hat{G})$ acting by

$$
(f \zeta g)\left(\chi, \chi^{\prime}\right)=f(\chi) \zeta\left(\chi, \chi^{\prime}\right) g\left(\chi^{\prime}\right) \text {. }
$$

The real Jordan sub-bimodule of this bimodule (cf. [17]) is generated by the constant function 1.

By arguing exactly as in [16], it follows that

$$
C \cong \Phi\left(L^{\infty}(G), \operatorname{Tr}\right) \cong L\left(\mathbb{F}_{\infty}\right) \otimes B(H)
$$

as claimed. 
The same proof works to show that

Theorem 5.2. Let $M=\Gamma(\sigma)$ as before and let $\phi$ be the free quasi-free state on $M$. Then the $G$ core of the $n$-fold free product $(M, \phi)^{* n}$, for any $n \geq 1$ or $n=+\infty$, is $L\left(\mathbb{F}_{\infty}\right) \otimes B(H)$.

Proposition 5.3. Let $(N, \theta)$ be a full factor with a G-state $\theta$. Assume that the compression of the $G$ core of $N$ to any of its finite projections can be embedded into $R^{\omega}$. Denote by $C$ the $G$-core of $(N, \theta) *\left(\Gamma\left(\nu_{\hat{G}}\right), \phi\right)$. Then $\delta(C)=+\infty$. In particular, $C$ is full.

Proof. Fix $p \in N \rtimes_{\sigma} G$ a projection of trace 1. By [17],

$$
\begin{aligned}
C & \cong\left(N \rtimes_{\sigma} G\right) *_{L(G)}\left(M \rtimes_{\sigma} G\right) \\
& \cong\left(N \rtimes_{\sigma} G\right) *_{L(G)} \Phi(L(G), \operatorname{Tr}) \cong \Phi\left(N \rtimes_{\sigma} G, \operatorname{Tr}\right) \\
& \cong\left(p\left(N \rtimes_{\sigma} G\right) p * L\left(\mathbb{F}_{\infty}\right) \otimes B(H),\right.
\end{aligned}
$$

the last isomorphism by [12] (see also [7]). Since by assumption $N \rtimes_{\sigma} G$ is embeddable in $R^{\omega}$, we get that $\delta(C)=+\infty$.

For the purpose of the next theorem, it is useful to make the following remark (which is probably folklore, but we sketch the proof nonetheless):

Proposition 5.4. The existence of an almost-periodic state is equivalent (for a separable full factor $M$ ) to the requirement that there be an almost-periodic weight.

Proof. We may of course restrict to the case that $M$ is of type $\mathrm{III}_{1}$. If there is an almost-periodic weight, then by Connes' results 4, $M \cong N \rtimes \Gamma$, where $N$ is a separable semi-finite von Neumann algebra, and $\Gamma$ is a countable discrete abelian group acting on $M$ by trace-scaling automorphisms $\alpha_{\gamma}$. There is a normal conditional expectation $E$ from $M$ onto $N$, given by integration over the group dual $\hat{\Gamma}$ of $\Gamma$. Let $\tau$ be the semifinite trace on $N$, and consider the semifinite weight $\psi=\tau \circ E$ on $M$. The modular group of $\psi$ is almost-periodic, and $\sigma^{\psi}$ can be extended to a compact completion $G=\hat{\Gamma}$ of $\mathbb{R}$. Moreover, $N$ is in the centralizer of $\psi$ and the restriction of $\psi$ to $N$ is semifinite $(=\tau)$. It follows that if $d \in N_{+}$ and $\tau(d)<+\infty$, then the modular group of the state $\phi=\psi\left(d^{1 / 2} \cdot d^{1 / 2}\right)$ is given by $x \mapsto \sigma_{t}^{\psi}\left(\beta_{t}(x)\right)$, where $\beta_{t}(x)=d^{i t} x d^{-i t}$. Note that $\sigma_{t}^{\psi}$ and $\beta_{s}$ commute for all $t$ and $s$. Now choose $d$ so that its spectral measure is atomic and supported on $\Gamma$ (one can in fact choose $d$ so that its spectrum is supported on the set $\left\{\lambda^{-k}\right\}_{k \geq 0}$ for any $\lambda \in \Gamma, \lambda \neq 1)$. Then $\beta_{t}(x)$ also extends to $G$, and so $(s, t) \mapsto \beta_{s} \circ \sigma_{t}^{\psi}$ extends to an action of $G \times G$. Restricting this action to the diagonal of $G \times G$, we find that the modular group of $\phi$ extends to $G$, and so $\phi$ is an almost-periodic state.

Theorem 5.5. There exists a continuum of mutually non-isomorphic free ArakiWoods factors, each having no almost-periodic weights.

Proof. It was shown in [17] that for each topology $\tau(\mu)$ as discussed above, there exists a free Araki-Woods factor whose $\tau$ invariant is exactly $\tau(\mu)$. Moreover, a factor $M$ has an almost-periodic weight iff the completion of $\mathbb{R}$ with respect to $\tau(\mu)$ is compact in that topology (hence $\mu$ is atomic, as then $\mu$ is supported on the Pontrjagin dual $\Gamma \subset \mathbb{R}$, where $\mathbb{R} \subset \hat{\Gamma}$ is the inclusion of $\mathbb{R}$ into its completion with respect to $\tau$ ). By Theorem 2.3 there exists a continuum of mutually non-equivalent topologies $\tau\left(\mu_{\lambda}\right)$, with $\mu_{\lambda}$ non-atomic. 
Theorem 5.6. There exist two non-isomorphic free Araki-Woods factors, which cannot be distinguished by their $\tau$ invariant.

Proof. Let $M_{1}$ be the free Araki-Woods factor associated to the representation of $\mathbb{R}$ with spectral measure $\delta_{1}+\delta_{-1}+\lambda$, where $\lambda$ denotes the Lebesgue measure on the additive group $\mathbb{R}$. Then by Theorem 5.1 and by Corollary 4.5 .

$$
\mathscr{C}\left(M_{1}\right) \supset \mathscr{C}_{\lambda} .
$$

Moreover, we have that $\tau\left(M_{1}\right)=\tau\left(\delta_{1}+\delta_{-1}+\lambda\right)$ is the usual topology on $\mathbb{R}[17$.

Let $M_{2}$ be the free Araki-Woods factor associated to the representation of $\mathbb{R}$ with spectral measure $\mu+\delta_{-1}+\delta_{1}$, where $\mu$ is as in Theorem 2.4. Then again $\tau\left(M_{2}\right)=\tau\left(\mu+\delta_{-1}+\delta_{1}\right)=\tau(\mu)$ is the usual topology on $\mathbb{R}$. Thus $\tau\left(M_{1}\right)=\tau\left(M_{2}\right)$. However,

$$
\mathscr{C}\left(M_{2}\right) \subset \mathscr{C}_{\sum \frac{1}{2^{n}}\left(\mu+\delta_{1}+\delta_{-1}\right)^{n}},
$$

so that

$$
\mathscr{C}\left(M_{2}\right) \cap \mathscr{C}_{\lambda}=\emptyset
$$

Hence

$$
\mathscr{C}\left(M_{2}\right) \neq \mathscr{C}\left(M_{1}\right)
$$

and so $M_{1}$ and $M_{2}$ are not isomorphic. In fact, going through the proof of Theorem 4.4, we see that the cores of $M_{1}$ and $M_{2}$ are not isomorphic (one has $\delta\left(M_{1}\right)>1$, $\left.\delta\left(M_{2}\right) \leq 1\right)$.

\section{ACKNOWLEDGEMENT}

This research was supported by an NSF postdoctoral fellowship, a Sloan Foundation fellowship, and NSF grant DMS-0102332. I thank MSRI and the organizers of the operator algebras program for their hospitality and for the encouraging atmosphere. I am also grateful to Professors N. Brown, T. Giordano, U. Haagerup, Y. Ueda and D.-V. Voiculescu for many useful discussions.

\section{REFERENCES}

1. L. Barnett, Free products of von Neumann algebras of type III, Proc. Amer. Math. Soc. 123 (1995), 543-553. MR 95c:46096

2. A. Connes, Correspondences, unpublished notes.

3. Une classification des facteurs de type III, Ann. scient. Éc. Norm. Sup. 6 (1973), 133-252. MR 49:5865

4. MR 50:10840

5. — Noncommutative geometry, Academic Press, 1994.

6. K. Dykema, Free products of finite dimensional and other von Neumann algebras with respect to non-tracial states, Free Probability (D.-V. Voiculescu, ed.), Fields Institute Communications, vol. 12, American Mathematical Society, 1997, pp. 41-88. MR 98c:46131

7. K. Dykema and F. Rădulescu, Compressions of free products of von Neumann algebras, Math. Ann. 316 (2000), no. 1, 61-82. MR 2001f:46100

8. C. C. Graham and O. C. McGehee, Essays in commutative harmonic analysis, SpringerVerlag, New York, 1979. MR 81d:43001

9. U. Haagerup, The standard form of von Neumann algebras, Math. Scand. 37 (1975), 271-283. MR 53:11387

10. E. Hewitt and K. A. Ross, Abstract harmonic analysis. Vol. I, second ed., Springer-Verlag, Berlin, 1979, Structure of topological groups, integration theory, group representations. MR 81k:43001

11. S. Popa, Correspondences, INCREST preprint, 1986. 
12. S. Popa and D. Shlyakhtenko, Universal properties of $L\left(\mathbb{F}_{\infty}\right)$ in subfactor theory, MSRI preprint 2000-032, to appear in Acta Math.

13. F. Rădulescu, A one parameter group of automorphisms of $L\left(\mathbb{F}_{\infty}\right) \otimes B(\mathscr{H})$ scaling the trace, C.R. Acad. Sci. Paris 314 (1992), no. 1, 1027-1032. MR 93i:46111

14. - A type $I I I_{\lambda}$ factor with core isomorphic to the von Neumann algebra of a free group, tensor $B(H)$, Recent advances in operator algebras (Orléans, 1992), no. 232, Astérisque, 1995, pp. 203-209. MR 97a:46083

15. D. Shlyakhtenko, Free quasi-free states, Pacific J. Math 177 (1997), 329-368. MR 98b:46086

16. Some applications of freeness with amalgamation, J. reine angew. Math. 500 (1998), 191-212. MR 99j:46079

17. _ A-valued semicircular systems, J. Func. Anal 166 (1999), 1-47. MR 2000j:46124

18. $375-425$.

19. D.-V. Voiculescu, The analogues of entropy and of Fisher's information measure in free probability theory II, Invent. Math. 118 (1994), 411-440. MR 96a:46117

20. - The analogues of entropy and of Fisher's information measure in free probability theory, III, Geometric and Functional Analysis 6 (1996), 172-199. MR 96m:46119

21. _ A strengthened asymptotic freeness result for random matrices with applications to free entropy, IMRN 1 (1998), 41-64. MR 2000d:46080

22. D.-V. Voiculescu, K. Dykema, and A. Nica, Free random variables, CRM monograph series, vol. 1, American Mathematical Society, 1992. MR 94c:46133

Department of Mathematics, University of California los Angeles, Los Angeles, CALIFORNia 90095

E-mail address: shlyakht@math.ucla.edu 